\section{Case Reports in Ophthalmology}

\title{
Topical Corticosteroid-Resolved Rubeosis Iridis with Neovascular Glaucoma Caused by Noninfectious Granulomatous Uveitis
}

\author{
Daisuke Sora Kei Takayama Manzo Taguchi Tomohito Sato \\ Yutaka Sakurai Takayuki Kanda Masaru Takeuchi \\ Department of Ophthalmology, National Defense Medical College, Tokorozawa, Japan
}

\section{Keywords}

Rubeosis · Topical corticosteroid · Uveitis · Neovascular glaucoma · Inflammation

\begin{abstract}
Purpose: We report a case of topical corticosteroid treatment-resolved rubeosis iridis with neovascular glaucoma (NVG) caused by noninfectious granulomatous uveitis. Case Report: A 61-year-old woman with left ocular pain and blurred vision was referred to our department. Visual acuity and intraocular pressure (IOP) were $20 / 60$ and $37 \mathrm{~mm} \mathrm{Hg}$ in the left eye, respectively. Inflammatory cells, hyphema, and rubeosis iridis were observed. All laboratory tests, including multiplex polymerase chain reaction for infection using aqueous humor, were negative, and there was neither retinal occlusive vasculitis nor retinal ischemia in the fundus. Our diagnosis was noninfectious granulomatous anterior uveitis-associated NVG. Topical corticosteroid treatment and anti-glaucoma agents resolved inflammation, rubeosis iridis, and NVG. IOP had decreased to $13 \mathrm{~mm} \mathrm{Hg}$ by 1 month of treatment, and no recurrence was observed. Conclusion: Topical corticosteroid could resolve rubeosis iridis and NVG in an eye with noninfectious anterior uveitis. The pathogenesis of NVG in eyes with uveitis is still unknown, but inflammation might have a part in angiogenesis. Anti-inflammatory treatment can be selected as the first choice for anterior uveitis-associated NVG.

(c) 2018 The Author(s)

Published by S. Karger AG, Basel
\end{abstract}




\section{Case Reports in Ophthalmology}

Case Rep Ophthalmol 2018;9:243-247

DOI: $10.1159 / 000488137$

(c) 2018 The Author(s). Published by S. Karger AG, Basel www.karger.com/cop

Sora et al.: Topical Corticosteroid-Resolved Rubeosis Iridis with Neovascular Glaucoma Caused by Noninfectious Granulomatous Uveitis

\section{Introduction}

Neovascular glaucoma (NVG) occurs when new fibrovascular tissue proliferates, including rubeosis iridis of the chamber angle, obstructs the trabecular meshwork, and produces peripheral anterior synechiae and progressive angle closure $[1,2]$. The increased intraocular pressure (IOP) is often difficult to control and frequently results in loss of vision [3]. NVG has been reported to occur in many ocular diseases, although most of these diseases have retinal ischemia as a common inciting factor [4]. In rare cases, uveitis causes rubeosis iridis and NVG without retinal ischemia $[2,5,6]$.

At present, a combination of an anti-glaucoma agent, retinal photocoagulation, intravitreal anti-vascular endothelial growth factor (VEGF) injection, or a surgical procedure is chosen as the first-line treatment for eyes with retinal ischemia-associated NVG [7, 8], although the outcomes are severe in most of the cases. In eyes with NVG caused by uveitis, anti-inflammatory treatment would be more effective. We report a case of topical corticosteroid treatment that resolved the rubeosis iridis and NVG in an eye with noninfectious anterior granulomatous uveitis.

\section{Case Report}

A 61-year-old woman with NVG was referred to our department with left ocular pain and blurred vision for a duration of 5 days. She had been treated for diabetes mellitus 4 years ago with hypoglycemic agents and for asthma 10 years ago with bronchodilators. At the first visit, the best-corrected visual acuity and IOP were 20/40 and $13 \mathrm{~mm} \mathrm{Hg}$ in the right eye and 20/60 and $37 \mathrm{~mm} \mathrm{Hg}$ in the left eye, respectively. The right eye showed no inflammation in the anterior segment and posterior segment. The left eye showed ciliary hyperemia, infiltrating cells, hyphemia, rubeosis iridis, and cataract in the anterior segment (Fig. $1 \mathrm{a}, \mathrm{b}$ ); however, vitreous opacity or retinal vasculitis was not observed in the posterior segment (Fig. 1c). Gonioscopy examination detected nodules and rubeosis on the trabecular meshwork in the left eye. Fluorescence angiography did not detect any retinal vasculitis or retinal ischemia caused by diabetes mellitus (Fig. 1d). Serum examination detected slight increases in blood glucose (201 mg/dL), HbA1c (6.8\%), and erythrocyte sedimentation rate $(17 \mathrm{~mm} / \mathrm{h})$. C-reactive protein, angiotensin-converting enzyme, and interleukin-2 receptor were within normal ranges. Bilateral hilar-mediastinal lymphadenopathy was not detected on chest X-ray. Serum herpes simplex virus (HSV) IgM, HSV IgG, cytomegalovirus (CMV) IgM, CMV IgG, and CMV antigen were negative, and a multiple broad-range polymerase chain reaction (PCR) test using anterior humor did not detect human herpes virus (HHV) 1-8, 16S rRNA, 28S rRNA, tuberculosis, or toxoplasmosis. We diagnosed the left eye as NVG caused by unknown anterior granulomatous uveitis, and topical corticosteroid $(0.1 \%$ betamethasone 6 times/day) and anti-glaucoma agents (0.005\% latanoprost 1 time/day, $0.1 \%$ brimonidine 2 times/day, $0.4 \%$ ripasudil hydrate 2 times/day, and $0.5 \%$ dorzolamide 3 times/day) were initiated. $\beta$ stimulant was not used because of asthma. On the next day, IOP was still high (28 $\mathrm{mm} \mathrm{Hg}$ ), and additional oral acetazolamide $500 \mathrm{mg}$ /day was initiated. After 1 week of treatment, inflammation and rubeosis iridis had diminished (Fig. 1e), and IOP had decreased to $20 \mathrm{~mm} \mathrm{Hg}$. After 1 month, inflammation and rubeosis iridis had completely resolved, and IOP had decreased to $13 \mathrm{~mm} \mathrm{Hg}$ (Fig. 1f). Gonioscopy also detected the regression of hyphema and rubeosis. Treatments were then tapered, and there was no recurrence for 1 year. 


\section{Case Reports in Ophthalmology}

Case Rep Ophthalmol 2018;9:243-247

DOI: 10.1159/000488137

(c) 2018 The Author(s). Published by S. Karger AG, Basel www.karger.com/cop

Sora et al.: Topical Corticosteroid-Resolved Rubeosis Iridis with Neovascular Glaucoma Caused by Noninfectious Granulomatous Uveitis

\section{Discussion}

NVG is a secondary ocular pathological condition resulting from a myriad of ocular and systemic conditions with retinal ischemia as a mediator in over 95\% of cases [2]. NVG is caused by the growth of a fibrovascular membrane secondary to a local angiogenetic stimulus over the trabecular meshwork obstructing aqueous outflow. The neovascularization process may occur on the iris, the iris and angle, or in the angle without concurrent iris involvement [9]. The growth of fibrovascular tissue, including vessels over the trabecular meshwork, will initially decrease aqueous outflow. Myofibroblasts, transiently present in new fibrovascular tissue, also proliferate and contract, thereby pulling the angle close. In the present case, rubeosis on the trabecular meshwork and rubeosis iridis were detected at the first visits, although no fibrovascular membrane existed. Since topical corticosteroid resolved rubeosis and IOP decreased to $13 \mathrm{~mm} \mathrm{Hg}$, trabecular meshwork would be saved from angle closure by a proliferative membrane.

Unilateral hyphemia, rubeosis iridis, and NVG have rarely occurred in eyes with HSVassociated uveitis $[4,10,11]$. To detect virus infection, multiplex and broad-range PCR has high sensitivity and specificity [12-14]. In this case, multiplex and broad-range PCR using aqueous humor were used, and HSV- or varicella zoster virus (HHV-3)-associated iridocyclitis was excluded.

VEGF plays an important role in the inflammatory process due to its promotion of angiogenesis and increased vascular formation [15]. The expression of VEGF is intimately linked to that of the major cytokines in the inflammatory cascade. Several inflammatory cytokines, including interleukin-1 $\beta$, tumor necrosis factor- $\alpha$, and transforming growth factor$\beta 2$, can upregulate VEGF production through various molecular pathways. The importance of VEGF in the development of uveitis, as well as its involvement in the inflammatory cascade, suggests that its inhibition may have a therapeutic potential when neovascularization occurs in the setting of uveitis [16]. While VEGF inhibition seems reasonable to treat secondary angiogenesis and increased vascular permeability in eyes with uveitis, anti-inflammatory treatment has a possible effect by inhibiting VEGF expression in eyes with uveitis, thus inhibiting the inflammatory cascade and inflammation-derived VEGF secretion.

\section{Conclusion}

Although this is a case report, topical corticosteroid can resolve rubeosis iridis and NVG in an eye with noninfectious anterior uveitis. The pathogenesis of NVG in eyes with uveitis is still unknown, but inflammation would have a part in angiogenesis. Anti-inflammatory treatment could be selected as the first choice for anterior uveitis-associated NVG.

\section{Statement of Ethics}

All procedures were done in accordance with the tenets of the Helsinki Declaration of 1964, as revised in 2013. Institutional review board approval was not required for this retrospective study involving a single case. We obtained informed consent from the patient. 


\section{Case Reports in Ophthalmology}

\begin{tabular}{l|l}
\hline Case Rep Ophthalmol 2018;9:243-247 \\
\hline DOI: 10.1159/000488137 & $\begin{array}{l}\text { @ 2018 The Author(s). Published by S. Karger AG, Basel } \\
\text { www.karger.com/cop }\end{array}$ \\
\hline
\end{tabular}

Sora et al.: Topical Corticosteroid-Resolved Rubeosis Iridis with Neovascular Glaucoma Caused by Noninfectious Granulomatous Uveitis

\section{Disclosure Statement}

The authors have no financial disclosures to make and no proprietary interests to disclose. There are no conflicts of interest for any of the authors.

\section{References}

1 Liao N, Li C, Jiang H, Fang A, Zhou S, Wang Q. Neovascular glaucoma: a retrospective review from a tertiary center in China. BMC Ophthalmol. 2016 Jan;16(1):14.

2 Hayreh SS. Neovascular glaucoma. Prog Retin Eye Res. 2007 Sep;26(5):470-85.

3 Kumari R, Zishan U, James M, Lucia K. Rubeotic glaucoma and what else should be worried about? Int Ophthalmol. 2012 Oct;32(5):461-2.

4 Fong DS, Raizman MB. Spontaneous hyphema associated with anterior uveitis. Br J Ophthalmol. 1993 Oct;77(10):635-8.

5 Fardeau C, Champion E, Massamba N, LeHoang P. Uveitic macular edema. Eye (Lond). 2016 Oct;30(10):1277-92.

6 Gaskin BJ, Danesh-Meyer HV. Neovascular glaucoma and sarcoidosis. Eye (Lond). 2005 May;19(5):599-601.

7 Qureshi K, Kashani S, Kelly SP. Intracameral bevacizumab for rubeotic glaucoma secondary to retinal vein occlusion. Int Ophthalmol. 2009 Dec;29(6):537-9.

8 Olmos LC, Lee RK. Medical and surgical treatment of neovascular glaucoma. Int Ophthalmol Clin. 2011;51(3):27-36.

9 Rodrigues GB, Abe RY, Zangalli C, Sodre SL, Donini FA, Costa DC et al. Neovascular glaucoma: a review. Int J Retina Vitreous. 2016 Nov;2(1):26.

10 Choi JA, Kim JE, Noh SJ, Kyoung Kim E, Park CK, Paik SY. Enhanced cytomegalovirus infection in human trabecular meshwork cells and its implication in glaucoma pathogenesis. Sci Rep. 2017 Feb; 7:43349.

11 Jap A, Chee SP. Viral anterior uveitis. Curr Opin Ophthalmol. 2011 Nov;22(6):483-8.

12 Sugita S, Shimizu N, Watanabe K, Ogawa M, Maruyama K, Usui N et al. Virological analysis in patients with human herpes virus 6-associated ocular inflammatory disorders. Invest Ophthalmol Vis Sci. 2012 Jul;53(8):4692-8.

13 Bharathi MJ, Murugan N, Rameshkumar G, Ramakrishnan R, Venugopal Reddy YC, Shivkumar C et al. Comparative evaluation of uniplex, nested, semi-nested, multiplex and nested multiplex PCR methods in the identification of microbial etiology of clinically suspected infectious endophthalmitis. Curr Eye Res. 2013 May;38(5):550-62.

14 Takayama K, Ogawa M, Mochizuki M, Takeuchi M. Cytomegalovirus retinitis in a patient with proliferative diabetes retinopathy. Ocul Immunol Inflamm. 2013 Jun;21(3):225-6.

15 Pożarowska D, Pożarowski P. The era of anti-vascular endothelial growth factor (VEGF) drugs in ophthalmology, VEGF and anti-VEGF therapy. Cent Eur J Immunol. 2016;41(3):311-6.

16 Gulati N, Forooghian F, Lieberman R, Jabs DA. Vascular endothelial growth factor inhibition in uveitis: a systematic review. Br J Ophthalmol. 2011 Feb;95(2):162-5. 


\section{Case Reports in Ophthalmology}
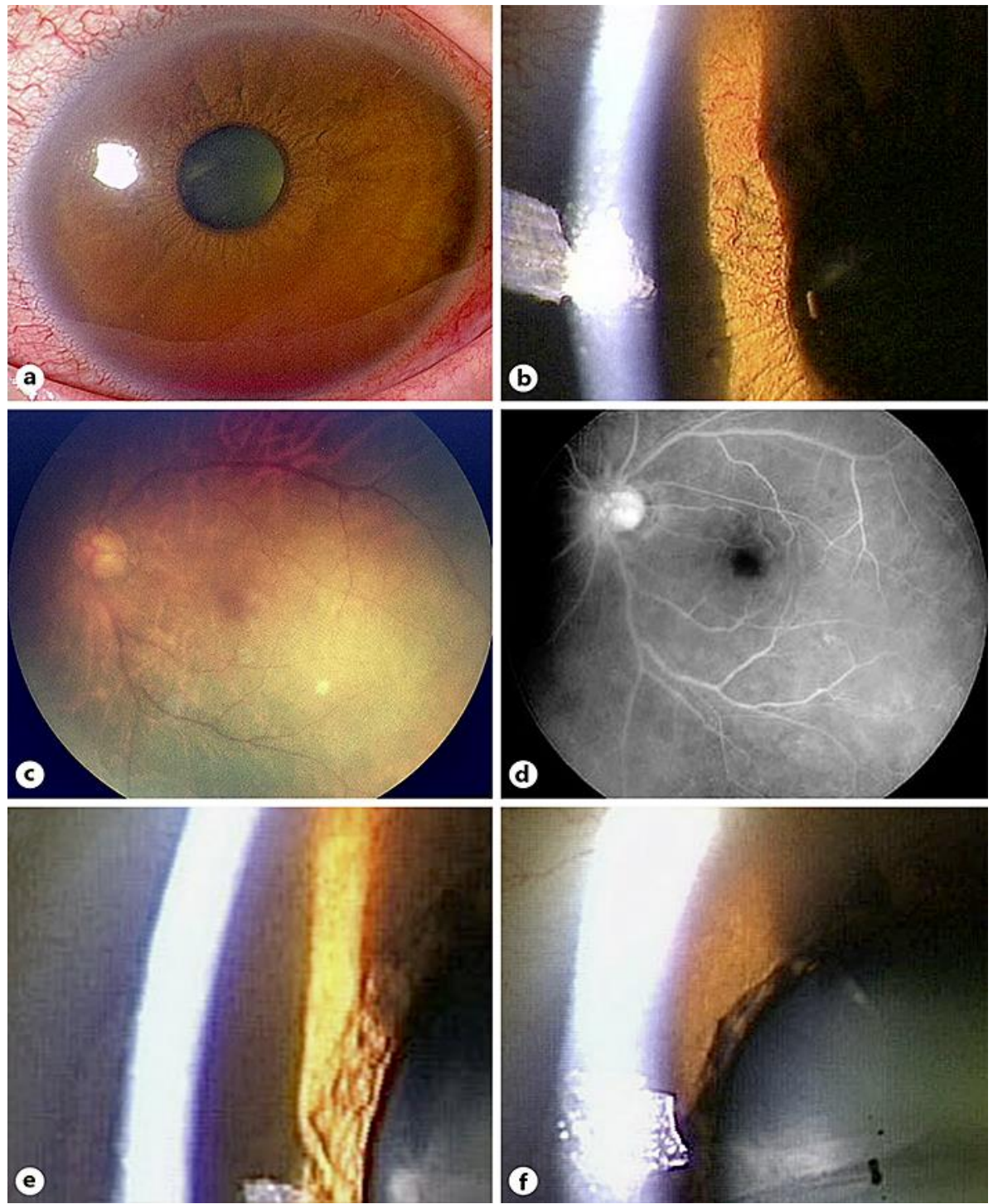

Fig. 1. Photos of the anterior segment and posterior segment and fluorescence angiography of the left eye. a, b Ciliary hyperemia, infiltrating cells, hyphema, rubeosis iridis, and cataract in the anterior segment. c Vitreous opacity or retinal vasculitis was not observed in the posterior segment. d Fluorescence angiography did not detect any retinal vasculitis or retinal ischemia. e, $\mathbf{f}$ Inflammation and rubeosis iridis were diminished after 1 week and had completely resolved after 1 month. 